\title{
Experimental Research on a Green Air-conditioning system based on Capacitive Deionization Regeneration Method
}

\author{
LI Xiu Wei ${ }^{1, ~ a ~, ~ C H E N G ~ F e n g ~}{ }^{1, b}$, DING Bo Qing ${ }^{1, c}$ and ZHAO Hui ${ }^{1, d}$ \\ ${ }^{1}$ College of Power Engineering, Nanjing University of Science and Technology, 210094, Nanjing, \\ PR China \\ agood3000best@163.com, ${ }^{\text {b }}$ fengcheng@njust.edu.cn, ${ }^{\text {c }}$ ding.boqing@qq.com, ${ }^{\mathrm{d}} 1846485264$ \\ @qq.com
}

Keywords: Energy; Air-conditioning; Regeneration; Performance.

Abstract. Green air-conditioning system is important for the development of low carbon society. Compared with vapour compression system, absorption system is more environment-friendly and can be driven by renewable energy. But the low performance impedes its development. The major reason lies in the energy waste in the thermal regeneration process. To improve, capacitive deionization (CDI) regeneration method is proposed. Driven by electric power, it regenerates absorbent with capacitors, thus avoids the waste heat and enhances the performance. To test the actual performance of the new method, experimental research has been conducted in this paper. The influence of some key parameters, like the concentration, has been investigated. The results expose the actual regeneration capacity and current efficiency under different working conditions. The actual COP attained 1.6 in the experiments, which indicates the new method could have better performance than that of the traditional thermal regeneration method.

\section{Introduction}

For today's society, sustainable development is challenged by energy crisis and climate change. It would not be improved unless we can reduce the emission from the combustion of fossil fuels, which is the main power supply to our world [1]. Energy conservation is an effective method to achieve this goal. This is especially true for buildings, which takes up more than $30 \%$ of the total energy for the society [2]. In buildings, air-conditioning system takes up more than $40 \%$ of the energy [3]. Therefore, an energy conservative air-conditioning is favorable as the present widely used vapour compression system consumes too much electric power and harms the environment. Absorption system is a greener choice, being environment-friendly and can be driven by renewable energy [4]. However, the performance of the absorption system is comparatively low. The major cause is the large energy waste in the traditional thermal regeneration process. Many progresses have been made to enhance the performance [5, 6], but no solution has been found to avoid the energy waste of heat. To solve this problem, we have proposed a capacitive deionization regeneration (CDI) method to replace the thermal method [7, 8]. Driven by electric power, the new method concentrates the absorbent solution in an electric field. With alternative deionization and regeneration process, both strong absorbent solution and pure water can be obtained. The new method avoids the energy waste and brings better performance. The energy recovery characteristic would make it better [7]. By preliminary analysis, it found the COP of the new method based system is higher than 5 under certain working conditions. Nevertheless, this result lacks the experimental data support. To test the new system, this paper presents the result of the experimental research. It exposes the influencing parameters and the actual performance of the CDI method.

\section{Principle of CDI Method}

CDI is an emerging water treatment technique that promotes the adsorption of ions in the electrochemical double layer (EDL) of a charged electrode surface by applying an electric potential, thus storing energy in the form of a capacitor and producing deionized water [9, 10]. Adsorbed ions are 
desorbed from the surface of the electrodes by eliminating the electric field, resulting in recovering a part of the energy used previously and producing a regenerated solution. Fig. 1 shows the process: In the deionization step, the electrodes are charged by applying a potential to water solution containing positive and negative charged ions, these ions are adsorbed on the electrodes, thereby producing deionized water at the outlet. In the regeneration step, a wash solution is circulated while the electrodes are depolarized, so that ions are desorbed from the electrodes and enter into the bulk of the solution, resulting in a stream of higher concentration.
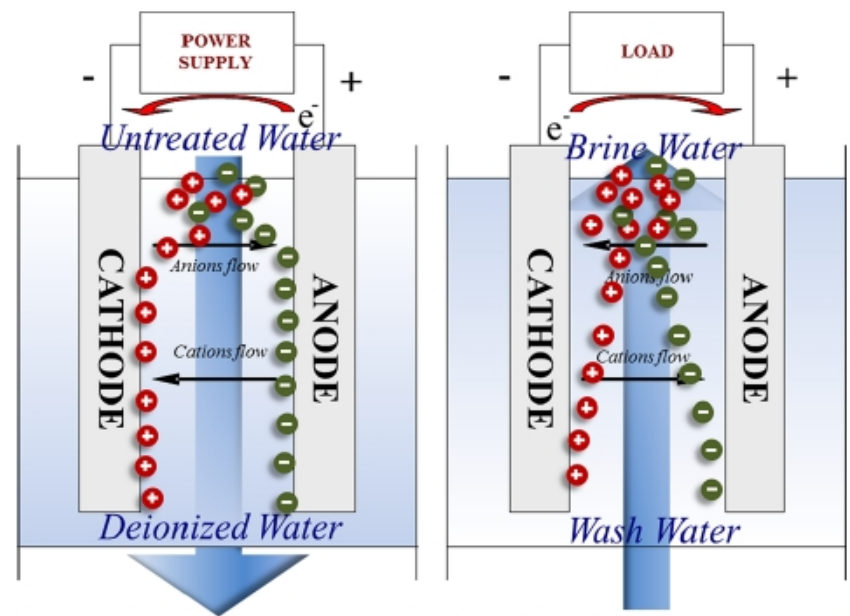

\section{DEIONIZATION}

REGENERATION

Fig. 1. Principle of the capacitive deionization process [9]

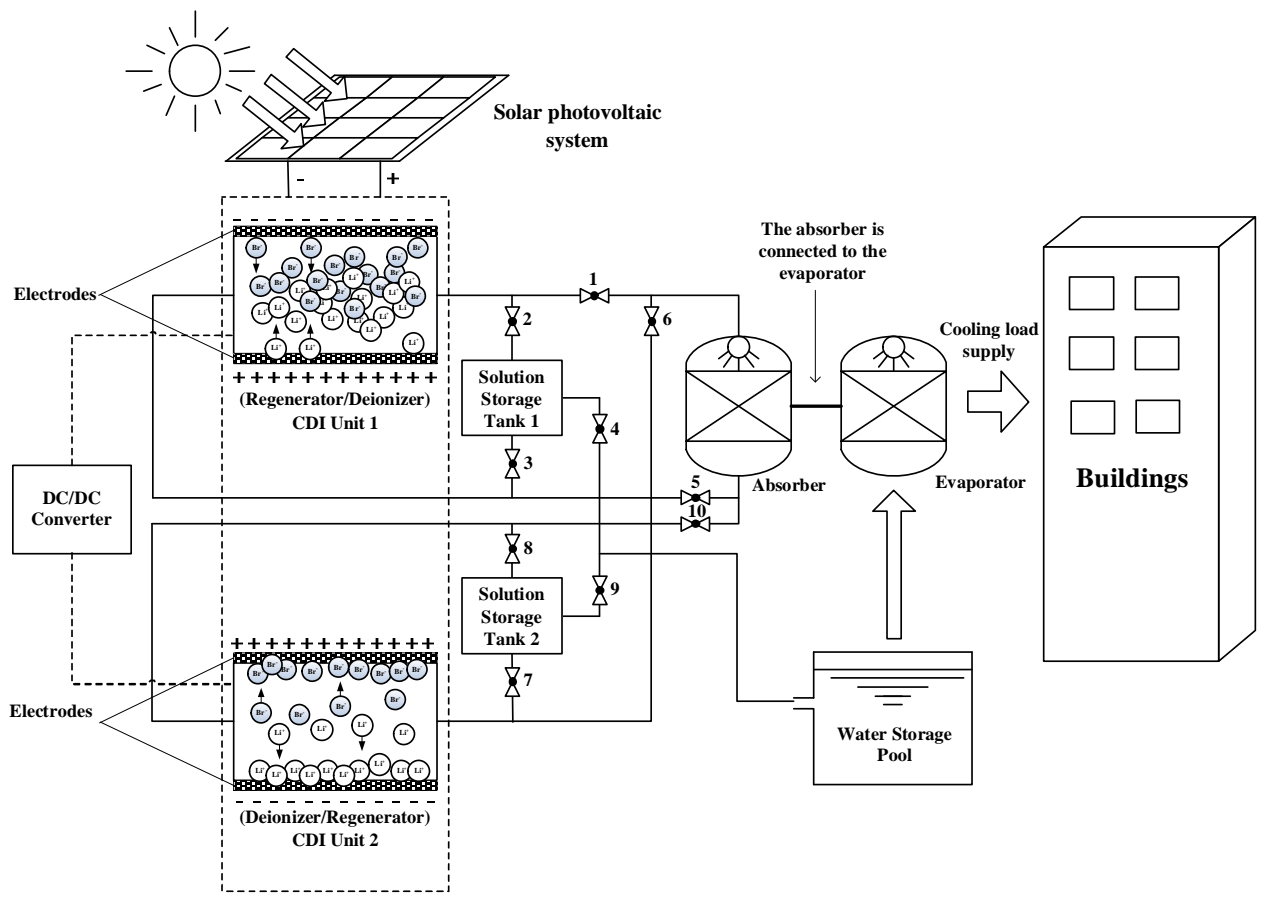

Fig. 2. Flow chart of the CDI regeneration air-conditioning system [7, 8]

\section{Method based Absorption Air-conditioning System}

The CDI regeneration based air-conditioning system is shown in Fig. 2 [7, 8]. The absorption and evaporation processes are same. The regeneration is completed with the joint work of two CDI units. By manipulating the valves, the two units alternate their roles as the regenerator and deionizer to 
produce both the strong absorbent solution and pure water. Meanwhile, the two units are connected with a DC/DC converter. It will transform the variable V-I curve into a constant current or constant voltage supply [9]. The regenerator also works as a discharging capacitor and applies the stored energy to deionize the solution passing through the deionizer.

\section{Experimental System testing the CDI Method}

CDI regeneration is the key part of the system and its performance must be tested. The experimental system is presented in Fig. 3 and Fig. 4. The system in Fig. 3 includes a deionization step and a regeneration step. First, the solution is pumped from the Salt Water Storage Tank 1 to the CDI unit and then stored in the Deionized Water Storage Tank 1. When the electrical conductivity of solution stops changing, the solution is pumped from the Deionized Water Storage Tank 2 to the CDI unit and stored in the Salt Water Storage Tank 2. The type of the pump was WXH-2201. The flow rate was regulated by a bypass-valve and measured with a flow meter, whose accuracy was $\pm 1 \%$. The concentration was recorded with a concentration meter, whose accuracy was $\pm 0.5 \%$. The voltage was detected with a multimeter, whose accuracy was $\pm 1 \%$. The energy consumed was recorded with a DC energy source with the accuracy of $\pm 0.5 \%$. The electrodes were made of the composite of carbon nanotube and active carbon. No energy recovery was available at present stage. With this experiment system, the actual performance of the CDI regeneration can be tested and the actual COP of the CDI based air-conditioning system can be derived from the experimental data.

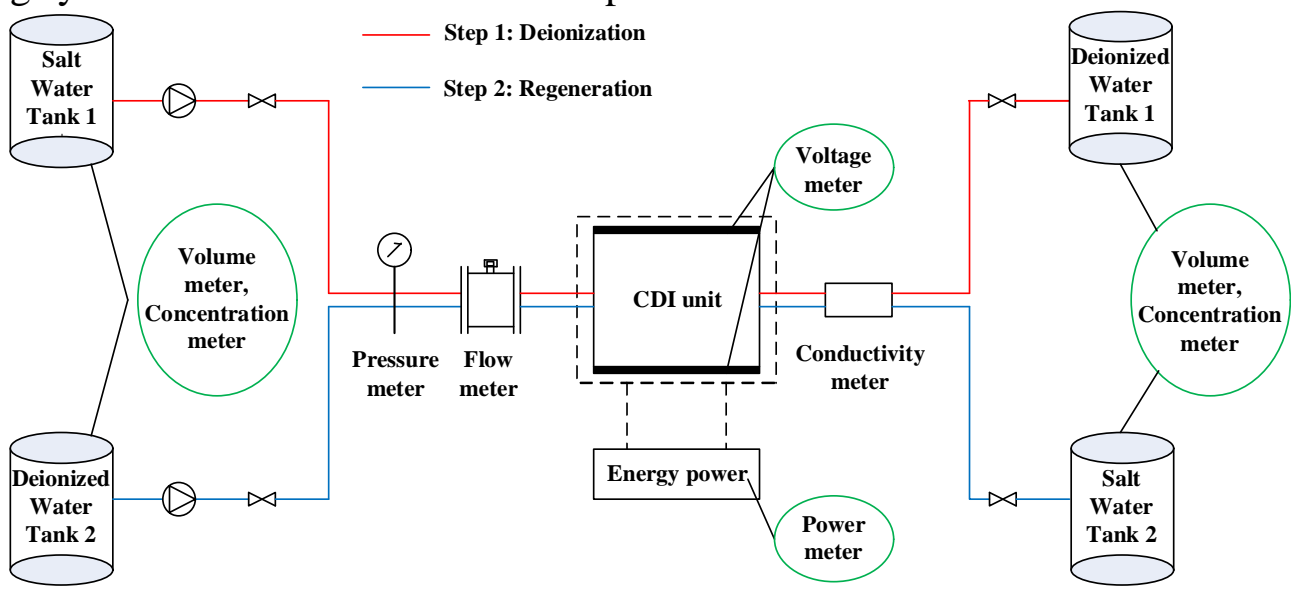

Fig. 3. Experimental system

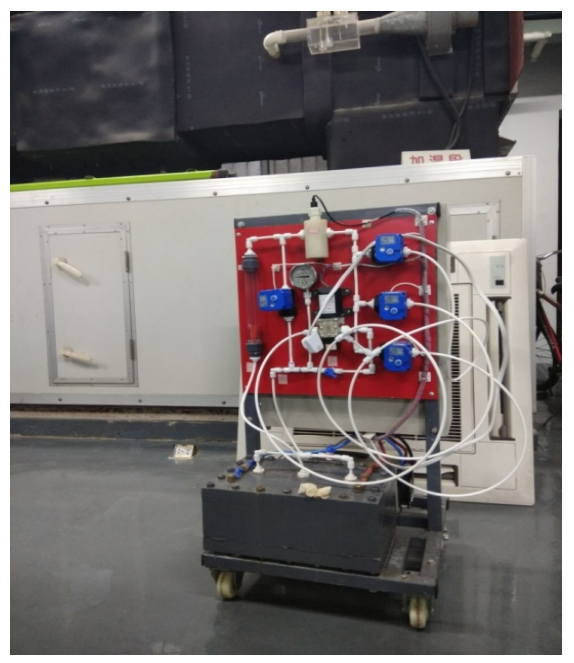

Fig. 4. CDI regenerator 


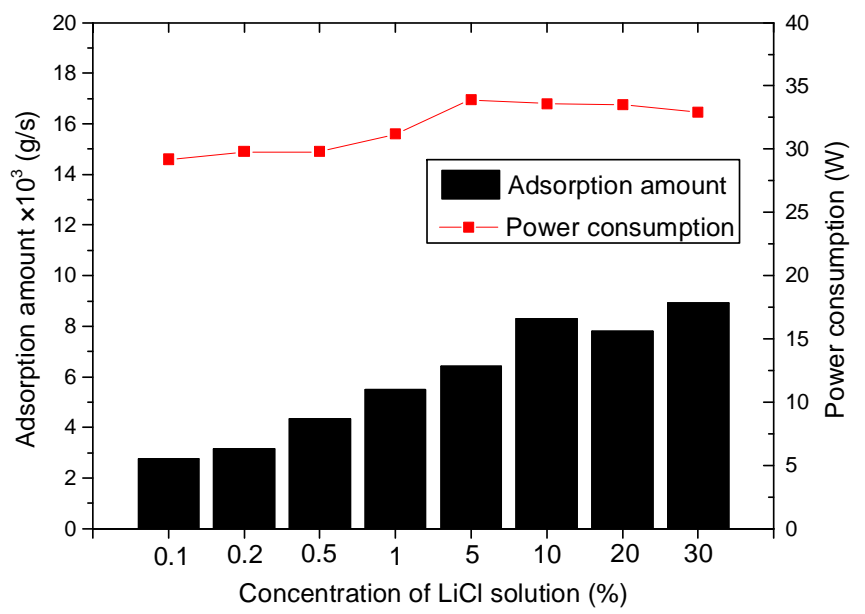

Fig. 5. Adsorption amount and power consumption with different absorbent concentration

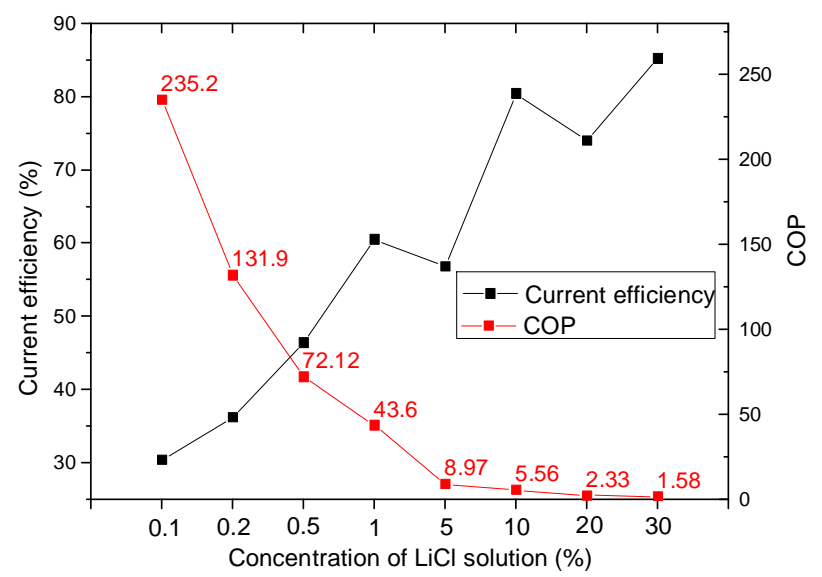

Fig. 6. Current efficiency and COP with different absorbent concentration

\section{Experimental Results}

For the CDI regeneration method, the mainly energy consumption is the power demand of deionization. With the amount of deionization (equal to that of regeneration), the cooling load can be calculated with the mass balance equations [7,8], and thus the actual COP is obtained as the ratio between the cooling load and the measured power. The experimental results are presented in Fig. 5 and Fig. 6 with $\mathrm{LiCl}$ solution as the absorbent. Fig. 5 shows the average deionization amount (adsorption amount in Fig. 5) and consumed power with different initial solution concentration, the applied voltage was maintained at $1.4 \mathrm{~V}$. In general, with the concentration increase of the absorbent solution, the adsorption amount increased, as well as the power consumption. More adsorption amount means more ions transfer, which is corresponding to higher energy demand. With the concentration above $10 \%$, the adsorption amount and consumed power increased slightly; it even decreased when the concentration was $20 \%$. It indicates an adsorption saturation state in the experimental system. The adsorption or deionization capacity is in proportion to the adsorption area of the electrodes. The result is useful for designing a regenerator of proper size for practical operation.

Based on Fig. 5, COP and the current efficiency can be calculated. The current efficiency is calculated with Eq. (5). Fig. 6 shows the results: The current efficiency generally increased with higher concentration. It rose rapidly with the concentration lower than $10 \%$. When the concentration was 
higher than $10 \%$, the current efficiency was around $80 \%$, seemed approaching its limitation. These data of actual current efficiency could be used for more practical and accurate analysis as it always assumes the efficiency is $50 \%$ in the present research $[7,8]$. Though the current efficiency has positive effect on performance, the COP decreased sharply with the concentration increase. Therefore, the concentration was the key factor influencing the performance. $\mathrm{As} \mathrm{LiCl}$ solution was the absorbent, a typical working range of concentration is $25 \% \sim 30 \%$. According to Fig. 6, the actual current efficiency was about $80 \%$ and the actual COP was about 2 1.6. This result is basically in accordance with that predicted by the models of CDI regeneration method $[7,8]$.

\section{Conclusions}

Experiments have been conducted to test the actual performance of the CDI regeneration air-conditioning system. The concentration of the regenerated solution is the most influential factor. The actual current efficiency increased with concentration increase, but the actual COP felt down quickly at higher concentration. It was around 2 in practical application, which is not very ideal compared with the vapour compression system. The experimental system did not adopt an energy recovery device. So the performance gets the potential to be better and it needs more works in the future.

\section{Acknowledgements}

This work was financially supported by This research was supported by the grants from the fund of National Natural Science Foundation of China under the contract No. 51676098, Natural Science Foundation of Jiangsu Province under the contract No. BK20160822 and BK20170095, the Fundamental Research Funds for the Central Universities under the contract No. 30917011327. These supports are gratefully acknowledged.

\section{References}

[1] F. Comino, M. R. de Adana, F. Peci: App. Therm. Eng. 131 (2018), p. 412-427.

[2] K. F. Fong, C. K. Lee: App. Therm. Eng. 127 (2017), p. 409-420.

[3] H. Huo, J. Shao, H. Huo: App. Therm. Eng. 124 (2017), p. 1159-1168.

[4] G. P. Kumar, R. Saravanan, A. Coronas: App. Therm. Eng. 132 (2018), p. 296-307.

[5] E. Akrami, I. Khazaee, A. Gholami: App. Therm. Eng. 129 (2018), p. 995-1001.

[6] B. Eisavi, S. Khalilarya, A. Chitsaz, M. A. Rosen: App. Therm. Eng. 129 (2018), p. 1219-1229.

[7] X. W. Li, X. S. Zhang, H. Wang, Z. Zhang: Energy Conversion and Management 114 (2016), p. $412-427$.

[8] X. W. Li, X. S. Zhang, H. Wang, Z. Zhang: Applied Energy 171 (2016), p. 405-414.

[9] Y. Qu, P. G. Campbell, L. Gu, J. M. Knipe, E. Dzenitis, J. G. Santiago, M. Stadermann: Desalination 400 (2016), p. 18-24.

[10] J. Kang, T. Kim, K. Jo, J. Yoon: Desalination 352 (2014), p. 52-57. 